\title{
One year of JISA-letter from the editors-in-chief
}

\author{
Fabio Kon - Gordon Blair
}

Published online: 9 December 2010

(C) The Brazilian Computer Society 2010

With this third issue, we reach the end of the first year of the Journal of Internet Services and Applications. As editorsin-chief, we have learned that creating a new journal and making it successful is a challenging task that requires a lot of hard work, but it is very worthwhile and rewarding. We remain committed to making JISA a key vehicle for the Internet research domain and are very happy with the great feedback and enthusiastic reception we received from both the academic and industrial communities.

We would like firstly to thank the authors of the 60 papers that have been submitted to JISA in this first year and the hard work of over 100 reviewers that helped us select the papers and provided valuable feedback to authors to improve their papers.

In the current issue, JISA features two invited papers from leading researchers in their fields, focusing on the most fundamental aspect of the Internet: the people that use it. The issue is then completed with two original research papers spontaneously submitted by their authors in the areas of Spam Filtering and Wireless Mesh Networks.

The first paper, "Human Aspects of Internet Services: Considering the Needs of Users and Providers" by Claudio Santos Pinhanez from IBM research, presents a classification of Internet Services according to the level of participation of people as providers and users of the service. It then discusses interface issues of Online Services, i.e., Internet Services in which humans perform a significant role both in providing and using the service. The paper uses the con-

\footnotetext{
F. Kon ( $\square)$

University of São Paulo, São Paulo, Brazil

e-mail: fabio.kon@ime.usp.br

G. Blair

Lancaster University, Lancaster, UK
}

ceptual framework of Service Science to challenge the notion that Internet services are computer-to-computer systems governed by machine protocols with no concern for human issues. We feel that this paper brings an important perspective to the community and we welcome follow up papers in this area.

The second paper, "A lightweight and extensible platform for processing personal information at global scale" by Michael Alexander Duller and Gustavo Alonso from ETH Zurich proposes a novel architecture for dissemination of personal information at a global scale that differs from currently used solutions by services such as Flickr, YouTube, Twitter, or Facebook. In their approach, personal data can be stored both in centralized data centers and in the idle storage resources of personal devices, enabling peer-to-peer exchange of information. Again, this is a core area for JISA and we welcome further papers related to managing personal information at a global scale. In this context, privacy issues play a key role that should be addressed by work in this area.

The third paper, "Spam Filtering: How the Dimensionality Reduction Affects the Accuracy of Naive Bayes Classifiers" by Tiago A. Almeida, Jurandy Almeida, and Akebo Yamakami from the University of Campinas addresses one of the major concerns of most Internet users for the past 10 years: spam. Spam filters based on Bayesian decision theory provide one of the best mechanisms to fight this digital plague caused by unethical businesses. In particular, Bayesian spam filters rely on term selection methods to address the problem of the high dimension of the feature space. However, it is not yet well understood how the application of these different methods affect the efficiency of spam filters. In this work, the authors have designed a set of experiments to evaluate the performance of many term selection techniques with several different models of Bayesian 
spam filters, obtaining statistically sound results. We sincerely hope that research results like this will help all of us by decreasing the amount of spam we need to read and by avoiding important messages to be mistakenly classified as spam.

Finally, the fourth paper, "Minimum Loss Multiplicative Routing Metrics for Wireless Mesh Networks" by Diego Passos, Célio Albuquerque, Miguel Elias M. Campista, Luís Henrique Costa, and Otto Carlos Duarte from UFF and UFRJ presents a study about the major routing metrics used in wireless mesh networks, which are low-cost, self-configurable, multihop networks. In wireless mesh networks, there is a subset of stationary nodes, called mesh routers, which are in charge of acting as routers for potentially mobile clients. Communication between this kind of ad hoc networks and other networks, such as the Internet, is performed by border mesh routers named mesh gateways. The authors have conducted comparative measurements over real mesh testbeds and also proposed a new multiplicative routing metric, which is shown to improve the network performance.
In 2011, we expect to receive even more submissions and look forward to firmly establishing JISA as a major outlet for work in Internet services and applications, but this is up to you, so we encourage our readers to submit their work for publication in JISA. In addition, in the next numbers, we will feature special issues on Cloud Computing, Network Virtualization, and Service Modeling and Design for the Future Internet.

Finally, feel free to contact the editors-in-chief if you have any requests, questions, or suggestions to improve the journal. Also do not hesitate to contact us if you are wondering whether your work would fit into the journal editorial line. We are eager to collaborate with the research community and work together with our authors and readers to make JISA an outstanding resource for our community.

Bangalore, India, December, 2010

Fabio Kon and Gordon Blair Editors-in-Chief 